\title{
Can stellar wobble in triple systems mimic a planet?
}

\author{
J. Schneider and J. Cabrera
}

Observatoire de Paris-Meudon, 92195 Meudon Cedex, France

e-mail: jean.schneider@obspm.fr

Received 17 May 2005 / Accepted 9 September 2005

\section{ABSTRACT}

The first extrasolar planets were detected by the measurement of the wobble of the parent star. This wobble leads to the periodic modulation of three observables: the radial velocity, the position on the sky and the time of arrival of periodic signals. We show that the same wobble, and therefore the same modulation of the three observables, can be due to the presence of a more distant binary stellar companion. Thus, the observation of the wobble does not, by itself, constitute a proof of a planet detection. In particular, astrometric confirmation of a wobble does not necessarily provide a sufficient proof of the existence of a planet candidate detected by radial velocity. Additional conditions, which we discuss here, must be fulfilled. We investigate the observed wobble for the planet candidates already detected and we find that, for each case, a wobble due to a binary stellar companion can be excluded.

However, for apparent Saturn-like planets in wide orbits, there may be an ambiguity in future detections, especially in spaceborne astrometric missions. We conclude that, in some cases, a definitive proof of the presence of a planet requires further observations such as direct imaging.

Key words. stars: planetary systems - astrometry - celestial mechanics

\section{Introduction}

The detection of the first extrasolar planets rests on an indirect method, namely the measurement of the reflex motion of the parent star. In cases where only the wobble is detected, one can ask whether the detection of radial velocity (RV) variations 1) are indeed due to a stellar wobble and not to other effects (such as stellar rotation or variable stellar activity); 2 ) are due to a planet and not to other dynamical effects; 3 ) that the companion is a planet (and not for instance a planetary mass black hole or strange matter object). In a few cases, the planet detection is confirmed by (or was preceded by) the detection of a transit of a planet, but the question of the planetary explanation of the wobble remains a priori open for the other candidates.

Here we consider the case where the wobble is real but due to the perturbation by a distant binary star first suggested by Schneider (1999).

\section{An approximation: restricted 3 body problem for hierachical systems}

Consider a triple hierachical system consisting of a binary system (with masses $M_{1}=M_{2}=M$; separation between components $2 a_{\mathrm{B}}$ ) and a third companion orbiting the center of mass of the system in a larger circular orbit (mass $M_{3}$; radius of the orbit $a_{\mathrm{A}}$ ). The perturbation caused by the binary system on the orbit of the third companion can imitate the perturbation caused by a planet around the latter star. In the appendix we have derived the equations of motion of such an approximation. The

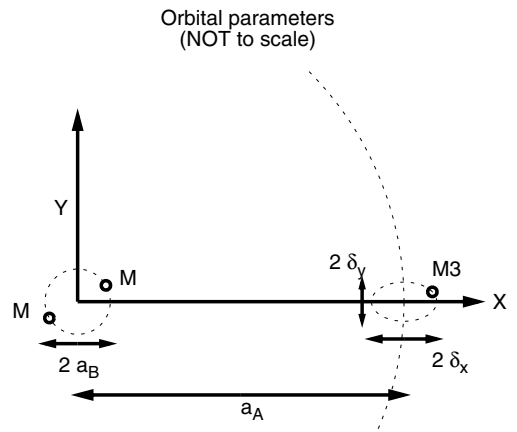

Fig. 1. Orbital elements of the system (not to scale).

result of the perturbation of the binary system is an elliptical periodic motion superimposed on the larger orbit of the third star. Studying this perturbation in the plane of the orbit of the third star, let the $X$ axis lay from the center of mass of the binary system to the target star, and the $Y$ axis perpendicular to it (see Fig. 1); the magnitude of this perturbation (see appendix) on both axes is:

$\delta_{x}=4.5 \frac{a_{\mathrm{B}}^{5}}{a_{\mathrm{A}}^{4}} \quad \delta_{y}=3 \frac{a_{\mathrm{B}}^{5}}{a_{\mathrm{A}}^{4}}$.

Our approximation takes as starting points the sizes of the orbits of the binary system $\left(a_{\mathrm{B}}\right)$ and the third star $\left(a_{\mathrm{A}}\right)$ together with the mass of the stars in the binary system $(M)$ and gives 


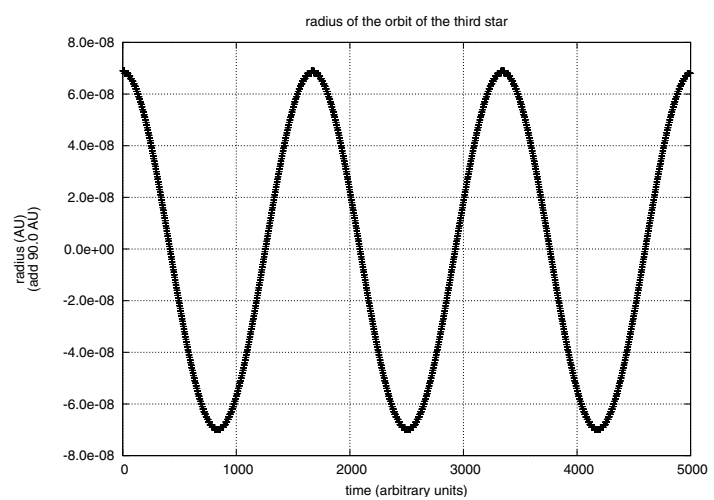

Fig. 2. Variation of the distance from the third star to the center of mass of the binary system.

the amplitude of the perturbation $\left(\delta_{x} ; \delta_{y}\right)$ which the binary system causes in the movement of the third star.

Stellar wobble and planetary companion produce, in principle, the same perturbation: a periodical elliptical motion which can be measured either by radial velocity or astrometry.

We have made simulations of such triple systems with the code KAPPA. Taking as initial parameters $a_{\mathrm{B}}, a_{\mathrm{A}}$ and $M$ (see Fig. 1) we can calculate the initial position and velocities for the three bodies which are required by the code (using Eqs. (A.1), (A.5) and (A.7)). Finally, we compare the results obtained in the simulation with those expected according to Eq. (A.6).

As an example, we choose $a_{\mathrm{B}}=1 \mathrm{AU} ; a_{\mathrm{A}}=90 \mathrm{AU} ; M=$ $1 M_{\odot}$. In Fig. 2 we see the radius of the orbit of the third star around the center of mass of the system. Over three years, it oscillates three times (half the period of the binary system, as expected). The radius fits very well to Eq. (A.6).

\section{Imitating a planet}

The perturbation of the binary system can induce a motion in the target star imitating a planet. In other words, we see a star with a periodic elliptical wobble. We suppose that this wobble is caused by the orbit of the star around the center of mass of the system star-planet. However, this wobble can be caused by a far binary system to which our target star is gravitationally linked. In our model, this motion will have the amplitude given by Eq. (1) and the following period:

$P_{*}=P_{\mathrm{pl}}=\frac{1}{2} P_{\text {binary }}=\frac{2 \pi a_{\mathrm{B}}^{3 / 2}}{\sqrt{G M}}$

where $M$ is the mass of each star of the binary system, $a_{\mathrm{B}}$ half the semi-major axis of the orbit of the binary system, as described previously. The subscripts $*$ and pl refer to the target star and the supposed planet respectively. Other parameters are:

$$
\begin{aligned}
& a_{\mathrm{pl}}=\left(\frac{G M_{*}}{4 \pi^{2}} P_{*}^{2}\right)^{1 / 3} \\
& e=\sqrt{1-\left(\frac{\delta_{y}}{\delta_{x}}\right)^{2}} \\
& M_{\mathrm{pl}}=M_{*} \frac{\delta_{x}}{a_{\mathrm{pl}}} .
\end{aligned}
$$

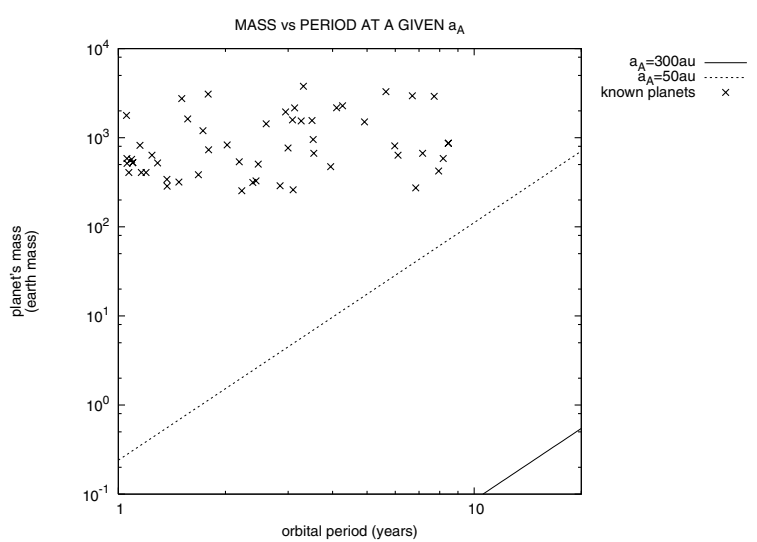

Fig. 3. The perturbation caused by the binary system in the target star will imitate a planet whose mass (for a given orbital period) lies on the straight lines, which represent distances from the target star to the center of mass of the binary system of 50 and $300 \mathrm{AU}$.

From these expressions, one can derive the mass $M$ required for each star of the hidden binary system to lead to the observed values for a pseudo-planet:

$M=(4.5)^{-3 / 5} M_{\mathrm{pl}}^{3 / 5} M_{*}^{2 / 5}\left(\frac{a_{\mathrm{A}}}{a_{\mathrm{pl}}}\right)^{12 / 5}$.

\section{Application to exoplanets detected by radial velocity}

One may wonder if the low amplitude wobble detected more than 150 stars (for a permanent update, see http://www.obspm. fr/planets) is due to a planet or to a more distant binary system. From the point of view of radial velocity measurements, a star is considered as single if there is no long term drift in its velocity curve.

In our model, we take a star supposed to be single with a planet companion but what is rather a star in a triple system. The absence of velocity drift imposes a minimum value for the distance of a hypothetical companion. $\gamma=G M / a_{\mathrm{A}}^{2}$ being the acceleration of the target star due to a companion at a distance $a_{\mathrm{A}}$, the velocity drift acquired over time $\Delta T$ is $\Delta V=\gamma \Delta T=G M / a_{\mathrm{A}}^{2} \Delta T$. The star is single if $\Delta V$ is smaller than the the observational limit. Taking from the last years of radial velocity surveys $\Delta V<10 \mathrm{~m} / \mathrm{s}$ and $\Delta T=5 \mathrm{yr}$, one gets, for $M=1 M_{\odot}, a_{\mathrm{A}}>300 \mathrm{pc}$.

From Eqs. (A.7), (2), (3) and (5) we obtain a relation between the mass of the supposed planet and the period of the wobble (with $M=M_{*}=1$ solar mass):

$M_{\text {planet }}\left(M_{\oplus}\right)=\left(\frac{35 \mathrm{AU}}{a_{\mathrm{A}}}\right)^{4}\left(\frac{P_{*}}{1 \text { year }}\right)^{8 / 3}$.

In Fig. 3 we represent this relation for $a_{\mathrm{A}}=300 \mathrm{AU}$ and $a_{\mathrm{A}}=$ $50 \mathrm{AU}$ together with the data for most of the known extrasolar planets. Clearly, none of the planets showed a risk of being a triple system, because the effect that a binary system causes is small. 
Table 1. Experimental data for planets in binary systems. The last column is the mass (calculated with 6) for each star of a hypothetical binary system which would cause in the target star the same wobble as the planet (see text). Data taken from Eggenberger et al. (2004) and from the Extrasolar Planets Encyclopaedia (http://www. obspm.fr/planets).

\begin{tabular}{lcccc}
\hline \hline Name & $a_{\mathrm{A}}(\mathrm{AU})^{a}$ & $a_{\mathrm{pl}}(\mathrm{AU})^{b}$ & $M_{\mathrm{pl}}\left(M_{J}\right)^{c}$ & $M\left(M_{\odot}\right)^{d}$ \\
\hline HD 40979 & 6400 & 0.811 & 3.32 & $3.0 \times 10^{7}$ \\
GL 777 A & 3000 & 4.8 & 1.33 & $3.6 \times 10^{4}$ \\
HD 80606 & 1200 & 0.469 & 3.90 & $2.0 \times 10^{6}$ \\
55 Cnc & 1065 & 0.115 & 0.84 & $1.8 \times 10^{7}$ \\
& & 0.24 & 0.21 & $1.4 \times 10^{6}$ \\
& & 5.9 & 4.05 & $3.7 \times 10^{3}$ \\
16 Cyg B & 850 & 1.6 & 1.5 & $2.8 \times 10^{4}$ \\
$v$ And & 750 & 0.83 & 2.11 & $1.3 \times 10^{5}$ \\
& & 2.50 & 4.61 & $1.5 \times 10^{4}$ \\
HD 178911 B & 640 & 0.32 & 6.292 & $1.6 \times 10^{6}$ \\
$\tau$ Boo & 240 & 0.05 & 4.08 & $1.1 \times 10^{7}$ \\
HD 195019 & 150 & 0.14 & 3.51 & $2.5 \times 10^{5}$ \\
HD 114762 & 130 & 0.35 & 11.03 & $3.9 \times 10^{4}$ \\
HD 19994 & 100 & 1.33 & 1.78 & $2.1 \times 10^{2}$ \\
$\gamma$ Ceph & 22 & 2.03 & 1.59 & $1.9 \times 10^{0}$ \\
Gl 86 & 20 & 0.11 & 4.0 & $3.5 \times 10^{3}$ \\
\hline
\end{tabular}

${ }^{a}$ Semimajor axis of the orbit of the binary stellar system in AU.

${ }^{b}$ Semimajor axis of the orbit of the planet in AU.

${ }^{c}$ Mass of the planet in Jupiter masses.

${ }^{d}$ Mass calculated with 6 for each star of the hypothetical binary system which would imitate the wobble of a planet.

\subsection{Planets in binary star systems}

There are presently around 15 planets detected in binary star systems (Eggenberger et al. 2004). One may wonder to what extent the companion of the target star hosting a planet is a binary system inducing a stellar wobble, imitating the effect of a planet.

Since the separation $a_{\mathrm{A}}$, the orbital parameters of the planet and the mass of the star (given its spectral type) are then known, the mass of the hypothetical binary is given by Eq. (6). In other words, if there is no planet and that there is a binary system perturbing the motion of the target star, we can calculate the mass of this system to see if this hypothesis is reliable. From the data of Eggenberger et al. (2004), Table 1 shows in each case the value for $M$ derived from Eq. (6).

The values found for $M$ are aberrant (except in the case of $\gamma \mathrm{Ceph}$ ); thus the corresponding stellar wobble cannot be explained as due to the binary nature of the companion star. In $\gamma$ Ceph the value of 2 solar masses is not aberrant; however, the mass found for the companion is 0.4 solar masses (Dvorak et al. 2003). Our model is consistent with a binary system of two solar masses each orbiting at 2.4 AU. That system has not been discovered and observational results do suggest this. We discart the existence of the triple system and accept the presence of a planet.

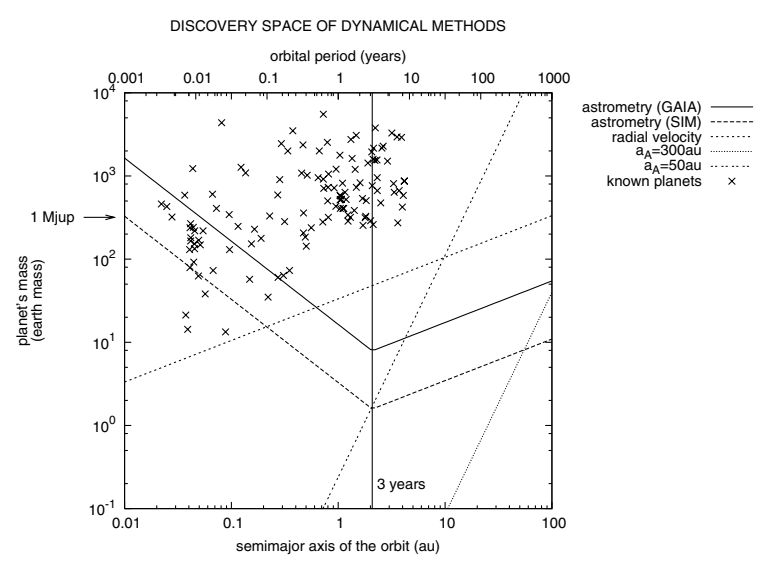

Fig. 4. Discovery space for GAIA, SIM and radial velocity missions, together with the expected perturbation caused by binary systems at 50 and $300 \mathrm{AU}$.

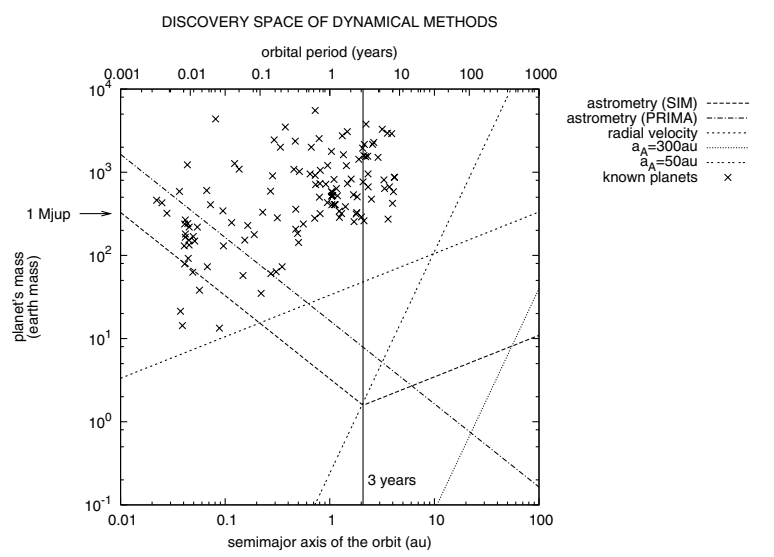

Fig. 5. Discovery space for SIM, PRIMA and radial velocity missions, together with the expected perturbation caused by binary systems at 50 and $300 \mathrm{AU}$.

\section{Application to future astrometric searches}

This may become more of an issue in the future, when more accurate measurements of stellar wobbles become available. Clearly, from Fig. 3, the wobble induced by a distant binary star mimics low mass planets on wide orbits. This configuration escapes planet detection by radial velocity but is well adapted to astrometric detection.

In Fig. 4 we represent the expected discovery space for two spatial missions: GAIA (Sozzetti et al. 2003) and SIM (Space Interferometry Mission, JPL). The constraints are the limiting resolution of 10 and 2 micro arcsec respectively and the lifetime of the missions: three years. Both will detect planets in regions still unexplored by radial velocity methods.

In Fig. 5 we compare the expected results for SIM and PRIMA (Phase-Referenced Imaging and Microarcsecond Astrometry at ESO VLTI). PRIMA has the same resolution as GAIA (10 micro arcsec) but is not constrained by the three years lifetime, having access to a low-mass long-period region where this stellar wobble effect will be more important.

The possibility of stellar wobble simulating a planet is small, however, for long periods and if the distance from the 
target star to the binary system $\left(a_{\mathrm{A}}\right)$ is small (e.g. $\left.50 \mathrm{AU}\right)$, these effects will have to be taken into account.

\section{Conclusion}

Stellar wobble by itself is not a proof that a planet has been detected. It is necessary to verify that no far binary star generates the wobble or to confirm the planet by transit or direct imaging observations. For the presently known planets, the explanation of a perturbing binary star can nevertheless be ruled out. But the sensitivity of GAIA, PRIMA and SIM is such that for some regions of the $\left(M_{\mathrm{pl}}, P_{\mathrm{pl}}\right)$ plane there can be an ambiguity between a true planet detected by astrometry and a wobble induced by a binary star.

Acknowledgements. We are grateful to R. Dvorak for the use of his 3-body numerical simulation software KAPPA.

This research has made use of the SIMBAD database, operated at CDS, Strasbourg, France.

\section{Appendix A}

The perturbation of the trajectory of a body in a triple system by a distant binary system is a classical issue in the 3-body problem (Roy 1979).

Consider a simplified triple hierachical system consisting of a binary system of two equal mass bodies $\left(M_{1}=M_{2}=M\right)$ in a circular orbit with radius $a_{\mathrm{B}}$ plus a third companion orbiting the center of mass of the binary system in a larger circular orbit with radius $a_{\mathrm{A}} \gg a_{\mathrm{B}}$.

Suppose that the motion of the binary system is not perturbed by the third body (let $M_{3}=0$ ) and let them move in the plane $z=0$. As we want a circular orbit with a given angular velocity $\omega$, we find that the equation of motion of the bodies $M_{1}$ and $M_{2}$ is:

$\vec{r}_{M_{1}}=a_{\mathrm{B}}(\cos \omega t \hat{i}+\sin \omega t \hat{j})$

$\vec{r}_{M_{2}}=-a_{\mathrm{B}}(\cos \omega t \hat{i}+\sin \omega t \hat{j})$

$\omega=\sqrt{\frac{2 G M}{\left(2 a_{\mathrm{B}}\right)^{3}}}=\sqrt{\frac{G M}{4 a_{\mathrm{B}}^{3}}}$.

The gravitational force per unit of mass at any point $\vec{r}=$ $(x, y, z)$ caused by these two bodies of mass $M$ is:

$\vec{F}=-G M\left(\frac{\overrightarrow{r_{1}}}{\left|\overrightarrow{r_{1}}\right|^{3}}+\frac{\overrightarrow{r_{2}}}{\left|\overrightarrow{r_{2}}\right|^{3}}\right)$

where $\vec{r}_{i}=\vec{r}-\vec{r}_{M_{i}} \quad i=1,2$.

To make the problem even simpler, we suppose that the motion of the third star is in the plane defined by the orbit of the binary system. In the plane we keep only the Cartesian coordinates $(x, y)$ or their polar equivalent $(r, \theta)$ with the identities for the unitary vectors:

$\hat{r}=\cos \theta \hat{i}+\sin \theta \hat{j}$

$\hat{\theta}=-\sin \theta \hat{i}+\cos \theta \hat{j}$

then we can separate Eq. (A.2) into the central force (in the direction of the center of mass of the binary system, parallel to $\hat{r}$ ) and the angular force (perpendicular to the former, parallel to $\hat{\theta}$ ):

$$
\begin{aligned}
\vec{r}_{1 / 2}= & \vec{r}-\vec{r}_{M_{1 / 2}} \\
= & {[r \cos \theta \hat{i}+r \sin \theta \hat{j}]-\left[ \pm a_{\mathrm{B}}(\cos \omega t \hat{i}+\sin \omega t \hat{j})\right] } \\
= & {\left[r \mp a_{\mathrm{B}} \cos (\omega t-\theta)\right] \hat{r} \mp a_{\mathrm{B}} \sin (\omega t-\theta) \hat{\theta} } \\
\left|\vec{r}_{1 / 2}\right|^{2}= & r^{2}+a_{\mathrm{B}}^{2} \mp 2 r a_{\mathrm{B}} \cos (\omega t-\theta) \\
\frac{1}{\left|\vec{r}_{1 / 2}^{3}\right|} \approx & \frac{1}{r^{3}}\left[1 \pm 3 \epsilon \cos (\omega t-\theta)+\frac{15}{2} \epsilon^{2} \cos ^{2}(\omega t-\theta)-\frac{3}{2} \epsilon^{2}\right] \\
\text { where } \epsilon \equiv & \frac{a_{\mathrm{B}}}{r} \ll 1 \\
\vec{F}^{2} \ddot{\vec{r}}= & -G M\left(\frac{r_{1}}{\left|r_{1}\right|^{3}}+\frac{\vec{r}_{2}}{\left|r_{2}\right|^{3}}\right) \\
\approx & -\frac{2 G M}{r^{2}}\left(\left[1+\frac{3}{4} \epsilon^{2}+\frac{9}{4} \epsilon^{2} \cos 2(\omega t-\theta)\right] \hat{r}\right. \\
& \left.\quad-\frac{3}{2} \epsilon^{2} \sin 2(\omega t-\theta) \hat{\theta}\right)
\end{aligned}
$$

correct to order 2 in $\epsilon$ and which is the same expression that we can find in Eq. (14.25) of Roy (1979) (under our assumption that the masses of the binary system are equal and the third mass is zero). As $\ddot{\vec{r}}=\left(\ddot{r}-r \dot{\theta}^{2}\right) \hat{r}+(2 \dot{r} \dot{\theta}+r \ddot{\theta}) \hat{\theta}$; we get:

$$
\begin{aligned}
& \ddot{r}-r \dot{\theta}^{2} \approx-\frac{2 G M}{r^{2}}\left[1+\frac{3}{4} \epsilon^{2}+\frac{9}{4} \epsilon^{2} \cos 2(\omega t-\theta)\right] \\
& 2 \dot{r} \dot{\theta}+r \ddot{\theta} \approx \frac{3 G M}{r^{2}} \epsilon^{2} \sin 2(\omega t-\theta) .
\end{aligned}
$$

As a solution to the system of differential Eqs. (A.4) we propose that the third body is in a circular orbit of radius $a_{\mathrm{A}}$ around the center of mass of the binary system with angular velocity $\Omega$ perturbed by another elliptical motion of semiaxes $\delta_{x}$ and $\delta_{y}$ both much smaller than $a_{\mathrm{A}}$. Thus the equation of motion of this body is, in rectangular coordinates:

$x(t)=a_{\mathrm{A}} \cos \Omega t+\delta_{x} \cos 2 \omega t$
$y(t)=a_{\mathrm{A}} \sin \Omega t-\delta_{y} \sin 2 \omega t$.

Notice:

1. This is not the most general solution. It is closer to a perturbation solution: these equations are solutions of the differential equation system (A.4).

2. The angular velocity of the perturbation is $-2 \omega$. As the two bodies of the binary system have the same mass, every half a revolution of the system the third body sees the same configuration of the binary system, in other words, the same configuration of the perturbation.

3. The Keplerian angular velocity goes as $a^{-1.5}$. As $a_{\mathrm{A}} \gg$ $a_{\mathrm{B}} \Rightarrow \omega \gg \Omega$. 
Taking this into account we arrive at the following equations to order one in the perturbation (that is, taking into account that $\left.\delta_{x} / a_{\mathrm{A}} \ll 1 ; \delta_{y} / a_{\mathrm{A}} \ll 1 ; \Omega / \omega \ll 1 ;\right)$ :

$$
\begin{gathered}
r \equiv \sqrt{x^{2}+y^{2}} \approx a_{\mathrm{A}}+\delta_{x} \cos 2 \omega t \\
\theta \equiv \arctan \frac{y}{x} \approx \Omega t \\
\dot{r} \equiv \frac{\mathrm{d}}{\mathrm{d} t} \sqrt{x^{2}+y^{2}} \approx-2 \omega \delta_{x} \sin 2 \omega t \\
\ddot{\theta} \equiv \frac{x \dot{y}-\dot{x} y}{r^{2}} \approx \Omega-2 \omega \frac{\delta_{y}}{a_{\mathrm{A}}} \cos 2 \omega t \\
\ddot{r} \equiv \frac{\mathrm{d}^{2}}{\mathrm{~d} t^{2}} \sqrt{x^{2}+y^{2}} \approx-4 \omega^{2} \delta_{x} \cos 2 \omega t \\
\ddot{\theta} \equiv \frac{\mathrm{d}}{\mathrm{d} t} \frac{x \dot{y}-\dot{x} y}{r^{2}} \approx 4 \omega^{2} \frac{\delta_{y}}{a_{\mathrm{A}}} \sin 2 \omega t .
\end{gathered}
$$

Introducing this solution (A.6) in the system of Eqs. (A.4) we obtain the following identities:

$a_{\mathrm{A}} \Omega^{2}+4 \omega^{2} \delta_{x} \cos 2 \omega t=\frac{2 G M}{a_{\mathrm{A}}^{2}}\left(1+\frac{3}{4} \epsilon^{2}+\frac{9}{4} \epsilon^{2} \cos 2 \omega t\right)$

$4 \omega^{2} \delta_{y} \sin 2 \omega t=\frac{3 G M}{a_{\mathrm{A}}^{2}} \epsilon^{2} \sin 2 \omega t$ from wich we get:

$$
\begin{aligned}
& \delta_{x}=4.5 \frac{a_{\mathrm{B}}^{5}}{a_{\mathrm{A}}^{4}} \quad \delta_{y}=3 \frac{a_{\mathrm{B}}^{5}}{a_{\mathrm{A}}^{4}} \\
& \Omega^{2}=\frac{2 G M}{a_{\mathrm{A}}^{3}}\left(1+0.75 \frac{a_{\mathrm{B}}^{2}}{a_{\mathrm{A}}^{2}}\right) .
\end{aligned}
$$

The mass of the binary system does not affect the amplitude of the perturbation but does affect the period $\left(T_{\text {wobble }} \sim(2 \omega)^{-1} \sim\right.$ $\left.M^{-0.5}\right)$. The angular velocity $\Omega$ is bigger than the Keplerian one, which means that for the same mass, the system is less bounded (the total energy $E=T-U$ is less negative).

\section{References}

Dvorak, R., Pilat-Lohinger, E., Funk, B., \& Freistetter, F. 2003, A\&A, 398, L1

Eggenberger A., Udry S., \& Mayor M. 2004, A\&A, 417, 353

Roy, A. E. 1979, in Orbital Motion (Bristol: Adam Hilger Ltd)

Schneider, J. 1999, American Astronomical Society, DPS meeting 31, No. 4, \# 5.02

Sozzetti A., Casertano S., Lattanzi M., \& Spagna A. 2003, in Proceeedings of the conference on Toward Other Earths: DARWIN/TPF and the Search for Extrasolar Terrestrial Planets (ESA SP-539, Noordwijk, Netherlands), 605 